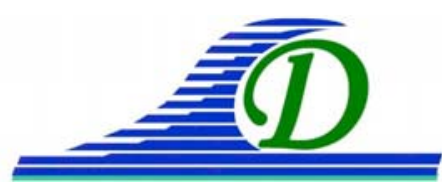
XIII İmes Journées Nationales Génie Côtier - Génie Civil
Dunkerque, 2-4 juillet 2014

DOI:10.5150/jngcgc.2014.031 @ Editions Paralia CFL

disponible en ligne - http://www.paralia.fr - available online

\title{
Trente-huit ans de rechargements sur la plage de Nice, Côte d'Azur : une synthèse statistique
}

\author{
Olivier COHEN ${ }^{1}$
}

\begin{abstract}
1. Université de la Nouvelle-Calédonie, Centre des Nouvelles Études sur le Pacifique (EA 4242), 145 avenue James Cook, BP R4, 98851 Nouméa, Nouvelle-Calédonie. olivier.cohen@univ-nc.nc
\end{abstract}

\section{Résumé :}

Le rivage de la ville de Nice, sur la Côte d'Azur en France, est bordé par un étroit cordon de galets de 4,5 km le long de la célèbre Promenade des Anglais. Cette plage évolue dans un contexte de basse énergie, mais connait cependant des problèmes d'érosion car elle est très artificialisée, est coupée de ses sources naturelles de sédiments et se situe au niveau d'une avant-côte très pentue. Depuis 1976, les services techniques de la mairie procèdent à des mesures de la largeur de la plage au niveau de 50 transects. Afin de lutter contre l'érosion et maintenir une largeur de plage suffisante pour les activités balnéaires, la mairie a déposé près de $600000 \mathrm{~m}^{3}$ de matériaux de rechargement depuis 38 ans. Nous disposons donc d'une base de données pour étudier l'évolution de cette plage et estimer l'efficacité de la politique de rechargement.

Mots-clés : Nice, Côte d'Azur, Cordon de galets, Mesures, Rechargements, Analyse statistique, Evolution, Erosion, Evaluation de la gestion.

\section{Introduction}

Comme celles des autres régions touristiques littorales, les plages de la Côte d'Azur ont une grande valeur patrimoniale. Elles sont indissociables de l'image de marque des stations balnéaires et des promenades associées (ex. Croisette à Cannes, Promenade des Anglais à Nice ; DEBIE, 1993). Ici, comme ailleurs, elles ont aussi un grand rôle dans la protection de zones densément urbanisées situées directement en arrière (ANTHONY, 1997 \& 2005 ; NORDSTROM, 2013). La commune seule regroupait 344064 habitants en 2011, l'agglomération en comptait 941490 en 2009. Elles sont fréquemment affectées par l'érosion côtière car elles sont coupées de leurs sources naturelles de sédiment et très artificialisées (ANTHONY \& COHEN, 1995 \& 1996 ; ANTHONY et al., 1998 ; GROUPE RAMOGE, 2002).

Pour tenter de stabiliser sa plage, la ville de Nice a opté depuis la fin des années 60 pour des rechargements. Cette technique est de plus en plus souvent utilisée pour les plages de sable (HAMM et al., 2002 ; HANSON et al., 2002 ; FINKL \& WALKER, 2005 ; VAN RIJN, 2011 ; COOKE et al., 2012). Les exemples de rechargement sur des cordons de galets sont plus rares (COHEN, 1996 ; BAWEDIN \& HOEBLICH, 2006). 


\section{Thème 2 - Dynamique sédimentaire}

Nous travaillons sur le cordon de galets de Nice depuis 20 ans (COHEN, 1993). Nos études se sont principalement appuyées sur des mesures de largeur de plage effectuées depuis 1976 et des volumes de rechargements que nous ont fournis les services techniques de la mairie. Grâce à des analyses de statistique descriptive et à des analyses de données, nous avons pu mettre en évidence les zones d'érosion et de progression de la plage et évaluer l'efficacité de la politique de rechargement (COHEN \& ANTHONY, 2007). Dans nos derniers travaux traitant des données jusqu'à 2005 (ANTHONY et al. 2011), nous avons montré que les apports massifs en galets (environ $558000 \mathrm{~m}^{3}$ ) avaient permis de stabiliser, puis d'élargir un peu la plage. Cependant, la majeure partie des apports a disparu vers les petits fonds très pentus. La question du devenir de la plage de Nice en cas de diminution ou d'arrêt des rechargements se posait donc. Déjà en 1996, FINKL avait abordé cette problématique pour les côtes sableuses du SE des USA : l'efficacité d'un rechargement dépend de la pérennité de sa source sédimentaire et financière.

\section{Présentation du site d'étude et de la politique de rechargements}

Nice se situe dans la région Provence Alpes Côte d'Azur, dans le département des Alpes Maritimes. La plage de Nice, longue de 4,5 km, constitue la partie nord-est de la Baie des Anges, le long de la Promenade des Anglais. Elle borde un arrière-pays élevé et pentu continuant directement en mer sans plateforme continentale ; la pente entre le rivage et l'isobathe $100 \mathrm{~m}$ varie de 20 à $80 \%$. Les profonds vallons entaillant les collines se prolongent en mer par des micro-canyons à quelques dizaines de mètres au large (figure 1). Le cordon est composé de galets de taille moyenne (5 à $10 \mathrm{~cm}$ ). Il évolue dans un contexte microtidal (50 cm en marée de vive-eau). Les vagues au large sont de basse énergie : de 2002 à 2014, 75\% des $H s \leq 0,7$ m, 99\% <1,9 m. Cependant des Hs de 3,7 m et $H_{\max }$ de 6,9 m ont été enregistrées lors de fortes tempêtes, ex. le 08/11/2011 (CEREMA, 2014). Les vagues les plus fréquentes viennent du SE à SW (65\%).

Dans le détail, le cordon présente un profil court (largeur moyenne $=28,17 \mathrm{~m}$ ), avec une faible dénivellation en haut de plage, marqué par de nettes bermes (figure 1). Cette plage est très artificialisée. Elle est adossée à un mur perré soutenant la Promenade des Anglais. Ces structures ont progressivement empiété sur le cordon originel dont la largeur historique est estimée, par les services municipaux, à une cinquantaine de mètres aux alentours de 1900 et une trentaine en 1930 (DUMASDELAGE et al., 2014). A l'ouest, la plateforme aéroportuaire constitue un obstacle infranchissable pour les apports de galets en provenance du fleuve le Var. Ceux des autres cours d'eau débouchant sur la plage (ex. Magnan et surtout Paillon), sont très limités également à cause de l'artificialisation de leurs cours aval et bassins-versants. A l'est, la plage se termine par le promontoire rocheux de Rauba Capeu. ANTHONY et al. (2011) ont montré que les départs de sédiments ne pouvaient donc se faire que vers le large et pas au-delà de ces obstacles. La plage est reprofilée chaque année : les bermes d’hiver sont lissées à la fin du printemps. Enfin, 16 épis en enrochement freinent le transit littoral. 


\section{XIII ${ }^{\text {èmes }}$ Journées Nationales Génie Côtier - Génie Civil \\ Dunkerque, 2-4 juillet 2014}

On trouve des configurations similaires dans la région, sur d'autres plages des Alpes Maritimes en France (ex. : Antibes, Cagnes-sur-Mer, Menton) et de Ligurie en Italie (ex. : Vintimille, Bordighera, San Remo; GROUPE RAMOGE, 2002) mais sans rechargements massifs comme à Nice.

Pour tenter de stabiliser sa plage, la ville de Nice a opté depuis la fin des années 60 pour des rechargements, mais les premières données recensées datent de 1976. Depuis, ce sont $582910 \mathrm{~m}^{3}$ qui ont été déposés, soit $128 \mathrm{~m}^{3}$ par mètre linéaire de plage. La réalisation de cette politique de gestion innovante pour l'époque a été en partie rendue possible par la disponibilité de matériaux gratuits, en provenance des grands chantiers de terrassement sur la commune (les collines niçoises sont constituées de poudingue, matrice sableuse contenant des galets) et du curage des petits cours d'eau (notamment le Paillon) débouchant sur la plage, dont la partie aval est aujourd'hui canalisée et souterraine (recouverte d'une dalle de béton urbanisée). Les rechargements sont amenés en fin d’hiver en général. Ils ont été irréguliers dans le temps. Sur la figure 2a, on voit que la plus grande partie a eu lieu de 1976 à $2000\left(525910 \mathrm{~m}^{3}\right)$. A partir de 2006, la mairie a dû acheter les matériaux à des carriers de l'arrière-pays $\left(80 € / \mathrm{m}^{3}\right)$. Depuis, ces apports ont principalement concerné la moitié est $\left(25000 \mathrm{~m}^{3}\right)$. La figure $2 \mathrm{~b}$ représente les volumes et les secteurs de dépôt.

A partir de 1976, les services techniques ont mis en place un réseau de suivi de la plage composé de 50 transects, espacés en $90 \mathrm{~m}$ en moyenne, le long desquels on mesure la largeur de la plage entre deux et quatre fois par an, en hiver, printemps et automne (figure 1). Nous disposons donc d'une base de données de 115 pas de temps de 1976 à 2013 sur 50 points de mesure, quasiment sans lacunes. Son analyse, croisée avec celle des rechargements, est riche d'enseignements. Nous en présentons ici certains résultats issus de traitements simples de statistique descriptive.

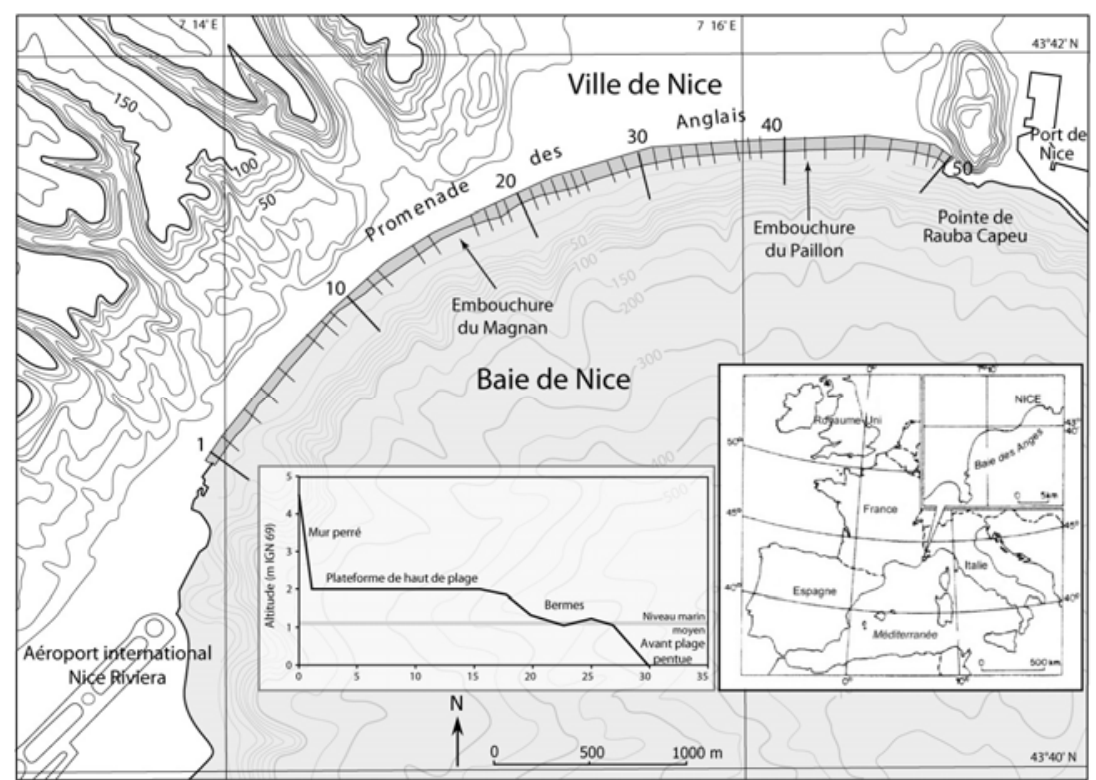

Figure 1. Localisation du site, réseau de mesure et profil caractéristique de la plage. 

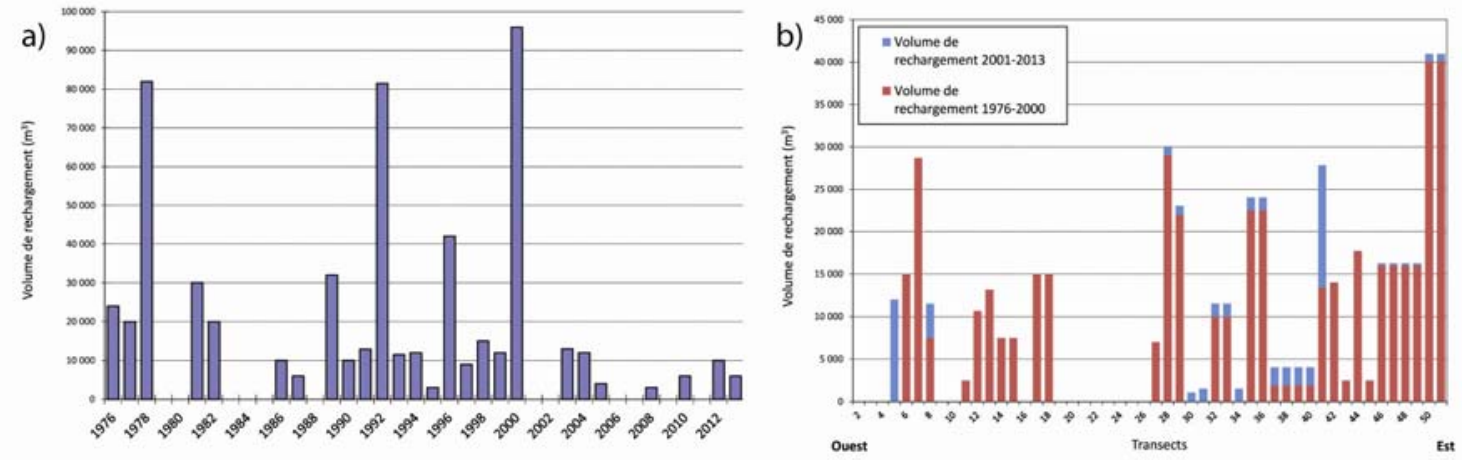

Figure 2. Les rechargements dans le temps et l'espace.

\section{Résultats}

La figure 3a montre l'évolution de la largeur moyenne annuelle de la plage de 1976 à 2013. Malgré une forte variabilité interannuelle, on peut mettre en évidence deux grandes périodes qu'une régression polynomiale d'ordre 3 permet de résumer : une phase de stabilisation (érosion de 1976 à 1981, puis léger élargissement jusqu’à 1998), puis une phase de forte érosion entamée à la fin des années 90 et qui se prolonge jusqu’à aujourd'hui. Elle correspond au quasi-arrêt des rechargements. Cette évolution générale cache bien sûr de fortes disparités spatiales de détail que fait apparaitre la figure 3b. On y remarque une forte variabilité sur de faibles distances avec une alternance rapide de secteurs plus ou moins larges que la moyenne générale de la plage (28 m ; trait horizontal en tiretés sur le graphique). Nous avons calculé les largeurs moyennes pour chaque transect de mesure de 1976 à 2013, de 1976 à 2000 et de 2001 à 2013. On s’aperçoit que, presque partout (36 sur 50 des transects, soit 72\%), les moyennes de la première phase sont supérieures et celles de la deuxième inférieures aux moyennes de toute la période d'étude. La répartition des rechargements ne semble pas toujours expliquer cette situation. Si l'est du cordon (T46 à 51) fait partie des secteurs les plus larges, c'est peut-être parce qu'il a bénéficié de très abondants apports $\left(211440 \mathrm{~m}^{3}\right)$. Néanmoins, d'autres secteurs rechargés (ex. T5 à 8, T27 à 29) ont une largeur plus étroite que la moyenne alors qu'ils ont reçus respectivement 67200 et $60130 \mathrm{~m}^{3}$ de galets.

Nous avons calculé les taux d'évolution pour chaque transect grâce à des régressions linéaires simples (figure 4). Les taux sur toute la durée d'étude varient entre plus et moins $20 \mathrm{~cm} / \mathrm{an}$ (trait en pointillés). On peut également distinguer une alternance de secteurs en érosion et en progression, mais leur "rythmicité" n’est pas aussi marquée que pour celle des largeurs. Ici aussi, nous avons déterminé les taux pour deux sous-périodes. Dans 76\% des cas, les taux de la seconde sont inférieurs à ceux de la première (la progression diminue ou l'érosion augmente). Cela est flagrant dans le $2^{\text {ème }}$ quart ouest (T14 à 23) et dans toute la partie est (T30 à 51) où l'on est passé d'une situation de quasi-stabilité voire de progression à une phase de nette érosion atteignant le mètre par an devant les galeries 


\section{XIII ${ }^{\text {èmes }}$ Journées Nationales Génie Côtier - Génie Civil \\ Dunkerque, 2-4 juillet 2014}

des Ponchettes. Comme pour la largeur moyenne, il est difficile de mettre en relation taux d'évolution et volumes de rechargement. Les rechargements abondants d'avant 2000 sur la partie la plus à l'est (T46 à 51) semblent avoir favorisé une progression. Leur quasiarrêt depuis 2001 se traduit par une forte érosion. Toutefois, d'autres secteurs (ex. T5 à 14), rechargés de 1976 à 2000 étaient en érosion et ceux sans rechargement de 2001 à 2013 sont en légère accrétion.
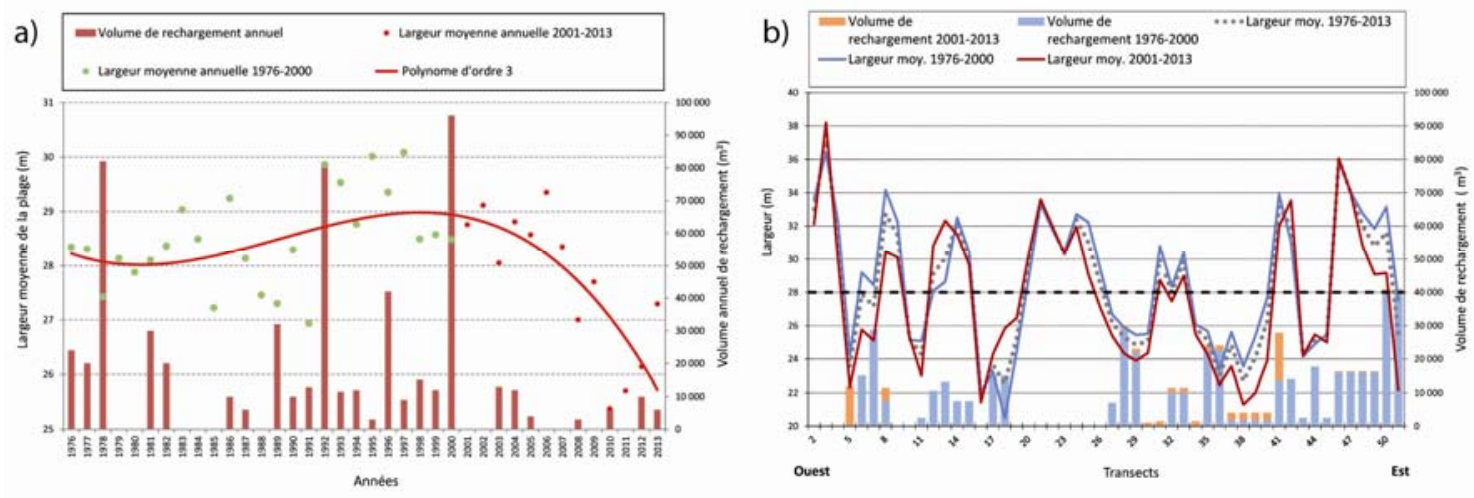

Figure 3. Largeurs moyennes dans le temps et dans l'espace.

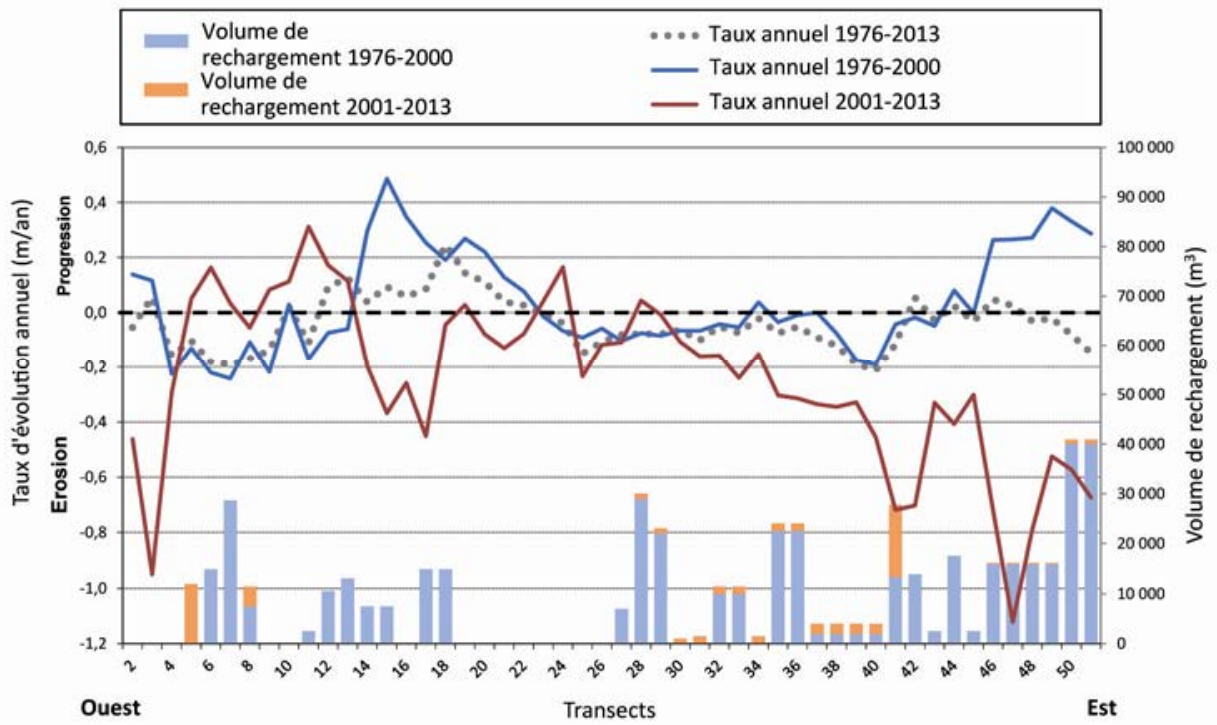

Figure 4. Comparaison des taux d'évolution des périodes 1976-2013, 1976-2000 et 2001-2013.

\section{Discussion et conclusions}

Si l'on peut dire que, globalement les rechargements ont permis de stabiliser la plage jusqu'à la fin des années 90 et que leur raréfaction a entrainé une phase d'érosion généralisée, dans le détail, il est difficile de déterminer le rôle des engraissements dans l'évolution des différents secteurs de la plage. Cela tient à plusieurs facteurs. 


\section{Thème 2 - Dynamique sédimentaire}

Les données tout d'abord : la base contient des largeurs de la plage émergée que l'on tente de mettre en relation avec des volumes. Des profils topographiques, notamment après les tempêtes, auraient été plus adaptés. C’est d'ailleurs ce type de mesure que les services techniques de la ville viennent de mettre en place (DUMASDELAGE et al., 2014). Les tableaux fournis par la mairie de Nice indiquent les lieux de dépôts des rechargements, mais ceux-ci sont ensuite étalés par les bulldozers et remaniés chaque année au printemps lors du reprofilage de la plage visant à atténuer les bermes formées en hiver. Une analyse fine des données saisonnières pourrait estimer l'impact de ces remaniements anthropiques de la plage.

Pour expliquer la variabilité spatiale de la largeur de la plage (figure 3), on pourrait avancer le rôle des épis sectorisant la plage où ils sont présents (14 épis sur 16 de T2 à T16). Ils créent des zones plus larges en amont-dérive littorale et plus étroites en aval dérive. Cela est visible sur les images aériennes. Mais on ne peut détecter l'influence de chaque épi dans sur le graphique. Sur le reste du cordon, il n’y a pas d'épis pour expliquer les variations de largeur.

Dans plusieurs publications (COHEN \& ANTHONY, 2007 ; ANTHONY et al., 2011), nous avions supposé que la bathymétrie de l'avant-côte, très pentue et présentant des micro-canyons jouant le rôle de piège à sédiment, devait avoir un rôle déterminant dans l'évolution de la plage. Cela a bien été démontré récemment par DUMASDELAGE et al. (2013 \& 2014). Grâce à des modèles hydrodynamiques (TOMAWAC, TELEMAC 2D et SISYPHE), ils ont mis en évidence une concentration de l'énergie des vagues sur les petits "caps" sous-marins séparant les canyons et, en fonction des directions de houles, des zones de convergence des courants de dérive. Dans ces travaux, le rôle des épis apparait également clairement. Une étude plus fine sur la corrélation entre les conditions hydrodynamiques modales et l'évolution du trait de côte serait intéressante à mener.

Pour élucider les impacts de la variabilité ou de la variation des conditions météomarines sur le rivage, on ne dispose que de peu d'éléments sur toute la période d'étude. Dans une analyse préliminaire, ANTHONY et al. (2011) font appel aux données ANEMOC (MORELLATO \& BENOIT, 2010). Cette analyse à l'échelle annuelle ne permet pas de détecter de variation de la hauteur significative des vagues en eau profonde et de la corréler avec l'évolution du cordon. A plus court terme, on pourra utiliser les données du houlographe installé depuis 2002 au large de l'aéroport de Nice (CEREMA, 2014). On pourra compléter l'étude en définissant l'historique des événements potentiellement érosifs (CHAVEROT et al., 2008).

En cette période où les rechargements sont devenus rares, il faut chercher à optimiser au mieux leur gestion. Pourra-t-on mettre en réserve les galets encore disponibles gratuitement parfois? Sans doute faudra-t-il mettre en question les reprofilages systématiques de la plage au printemps, notamment des étalements vers le bas de plage pour gagner en largeur, qui provoquent des pertes vers le large (ANTHONY et al., 


\section{XIII ${ }^{\text {èmes }}$ Journées Nationales Génie Côtier - Génie Civil \\ Dunkerque, 2-4 juillet 2014}

2011). Des études morphodynamiques visant à évaluer la stabilité des dépôts et les réactions du cordon aux entrées d’énergie seraient les bienvenues.

\section{Références bibliographiques}

ANTHONY E.J. (1997). The status of beaches and shoreline development options on the French Riviera: a perspective and a prognosis. Journal of Coastal Conservation, Vol. 3, pp 169-178. http://dx.doi.org/10.1007/BF02905242

ANTHONY E.J. (2005). Beach erosion. In M. L. Schwartz (ed.), Encyclopedia of Coastal Science, Springer, Dordrecht, pp 140-145.

ANTHONY E.J., COHEN O. (1995). Nourishment solutions to the problem of beach erosion in France : the case of the French Riviera. In HEALY AND DOODY (eds), Directions in european coastal management, Samara Publishing Ltd, Cardigan, pp 199-206. http://dx.doi.org/10.1002/(SICI)1099-1719(200005)8:2<121::AID-SD134>3.0.CO;2-6

ANTHONY E.J., COHEN O. (1996). Organisation et déstabilisation morphodynamiques des plages de galets de la Baie des Anges, Côte d'Azur. Revue d'Analyse Spatiale Quantitative et Appliquée, pp 38-39, Nice, pp 29-38.

ANTHONY E.J., COHEN O., SABATIER F. (2011). Chronic offshore loss of nourishment on Nice beach, French Riviera: a case of over-nourishment of a steep beach? Coastal Engineering, Vol. 58, pp 374-383. http://dx.doi.org/10.1016/j.coastaleng.2010.11.001

ANTHONY E.J., DUBAR M., COHEN O. (1998). Les cordons de galets de la Baie des Anges: histoire environnementale et stratigraphie ; évolution morphodynamique récente en réponse à des aménagements. Géomorphologie : relief, processus, environnement, $n^{\circ} 2$, pp 167-188. http://dx.doi.org/10.3406/morfo.1998.953

BAWEDIN V., HOEBLICH J-M. (2006) Les Bas-Champs de Cayeux (Somme, France) : vers une gestion intégrée ? VertigO - la revue électronique en sciences de l'environnement, Vol. 7(3). http://dx.doi.org/10.4000/vertigo.1910

CEREMA, 2014. Centre d'Archivage National de Données de Houle In Situ (CANDHIS). Données du houlographe de Nice. Disponible en ligne sur URL: http://candhis.cetmef.developpement-durable.gouv.fr/publications/00601/astx_00601_2.pdf

CHAVEROT, S., HÉQUETTE, A., COHEN O., 2008. Changes in storminess and shoreline evolution along the northern coast of France during the second half of the 20th century. Zeitschrift für Geomorphologie, 52, 3, 1-20. http://dx.doi.org/10.1127/03728854/2008/0052S3-0001

COHEN O. (1993). Stabilisation d'un cordon de galets par des rechargements; l'exemple de la plage de Nice, Côte d'Azur. Mémoire de DEA de géographie, Université de Nice Sophia-Antipolis; 50 p.

COHEN O. (1996). Approche méthodologique à l'étude des littoraux en Méditerranée française : exemples sur la Côte d'Azur. Thèse de doctorat en géographie, Université du Littoral Côte d’Opale, Dunkerque, 159 p. + annexes. 
COHEN O., ANTHONY E.J. (2007). Gravel beach erosion and nourishment in Nice, French Riviera. Méditerranée, Vol. 108, pp 98-103. http://dx.doi.org/10.4000/mediterranee.182 COOKE B.C., JONES A.R., GOODWIN I.D., BISHOP M.J. (2012) Nourishment practices on Australian sandy beaches: a review. Journal of Environmental Management, Vol. 113, pp 319-327. http://dx.doi.org/10.1016/j.jenvman.2012.09.025

DEBIE, F. (1993). Une forme urbaine du premier âge touristique: les promenades littorales. Mappemonde 1/1993, pp 32-37. Disponible en ligne sur URL: http://www.mgm.fr/PUB/Mappemonde/M193/PROMENAD.pdf

DUMASDELAGE R., DELESTRE O., CLAMOND D., BONNIN A., CERUTI P., MORETTI M., GOURBESVILLE P. (2013). Modeling of the erosion phenomena on Nice shoreface and the impact of coastal structures. Proceeding IAHR 2013, Chengdu, 7 p.

DUMASDELAGE R., DELESTRE O., CLAMOND D., BONNIN A., MORETTI M., CERUTI P., GOURBESVILLE P. (2014). Numerical modeling of the erosion phenomena on Nice shoreface using TELEMAC system. Proceeding IAHR 2014, Porto, 9 p.

FINKL C.W. (1996). What might happen to America's shoreline if artificial beach nourishment is curtailed: A prognosis for southeastern Florida and other sandy regions along regressive coasts. Journal of Coastal Research, Vol. 12, pp iii-ix. Disponible en ligne sur URL : http://journals.fcla.edu/jcr/article/view/79794/77062

FINKL C.W., WALKER H.J. (2005), Beach nourishment, In M. L. SCHWARTZ (ed.), Encyclopedia of Coastal Science, Springer, Dordrecht, pp 147-161. http://dx.doi.org/10.1007/978-94-017-0099-3_1

GROUPE RAMOGE (2002). La défense des plages contre l'érosion dans la zone RAMOGE. Guide à l'attention des maîtres d'ouvrages. Monaco, 25 p. Disponible en ligne sur URL : http://www.ramoge.org/Documents/documents\%20ramoge/ramoge_erosion_plages.pdf

HAMM L., CAPOBIANCO M., DETTE H.H., LECHUGA A., SPANHOFF R., STIVE M.J.F (2002). A summary of European experience with shore nourishment. Coastal Engineering, Vol. 47, pp 237-264. http://dx.doi.org/10.1016/S0378-3839(02)00127-8

HANSON H., BRAMPTON A., CAPOBIANCO M., DETTE H.H., HAMM L., LAUSTRUP C., LECHUGA A., SPANHOFF R. (2002). Beach nourishment projects, practices, and objectives - a European overview. Coastal Engineering, Vol. 47, p. 81111. http://dx.doi.org/10.1016/S0378-3839(02)00122-9

MORELLATO D., BENOIT M. (2010). Constitution d'une base de données d'états de mer le long des côtes françaises méditerranéennes par simulations rétrospectives couvrant la période 1979-2008. Revue Paralia, Vol. 3, pp 5.1-5.12. http://dx.doi.org/10.5150/revue-paralia.2010.005

NORDSTROM K.F. (2013). Developed coasts. In: Shroder J. (Editor in Chief), Sherman D.J. (Ed.), Treatise on Geomorphology. Academic Press, San Diego, CA, Vol. 10, Coastal Geomorphology, pp 392-416. http://dx.doi.org/10.1016/B978-0-12-374739-6.00292-X VAN RIJN L.C. (2011). Coastal erosion and control. Ocean \& Coastal Management, Vol. 54(12), pp 867-887. http://dx.doi.org/10.1016/j.ocecoaman.2011.05.004 